\title{
Space charge and resistive wall impedance computation in the frequency domain using the finite element method
}

\author{
Uwe Niedermayer, ${ }^{1, *}$ Oliver Boine-Frankenheim, ${ }^{1,2}$ and Herbert De Gersem ${ }^{1}$ \\ ${ }^{1}$ Institut für Theorie elektromagnetischer Felder, Technische Universität Darmstadt, \\ Schlossgartenstr. 8, D-64289 Darmstadt, Germany \\ ${ }^{2}$ GSI Helmholtzzentrum für Schwerionenforschung, Planckstr. 1, D-64291 Darmstadt, Germany
}

(Received 9 December 2014; published 26 March 2015)

\begin{abstract}
A two-dimensional finite element beam coupling impedance solver for arbitrary transverse geometries and material distribution is presented. The solver is based on open source software and is applicable to space charge and resistive wall longitudinal and transverse impedances. The frequency and the beam velocity can be chosen arbitrarily and also dispersively lossy materials are supported. Space charge impedance, a thin resistive beam pipe, a ferrite ring, and a carbon collimator are presented as application examples.
\end{abstract}

DOI: 10.1103/PhysRevSTAB.18.032001

PACS numbers: 41.75.Ak, 41.60.-m, 41.75.Lx

\section{INTRODUCTION}

The concept of beam coupling impedance describes the electromagnetic interaction of a particle beam with the surrounding equipment in the frequency domain (see, e.g., $[1,2])$. Particularly in circular accelerators, the frequency domain description is advantageous. For modern synchrotrons of large circumference, increasingly lower frequencies become important. Also, due to shorter bunch lengths, one has to focus on higher frequencies. For the estimation of coherent instability thresholds, an impedance model of the machine, such as the one presented in [3], is required. On the one hand, at high frequencies, time domain methods such as finite integration technique (FIT) or finite differences time domain (FDTD) are very efficient (see, e.g., [4,5] and references therein) and commercial codes are available. On the other hand, at low and medium frequencies, involved geometry approximations together with analytic calculations are widely used. Examples are [6,7] and the Mathematica ${ }^{\circledR}$ [8] script Rewall [9] developed by Mounet et al. at CERN. Numerical impedance computations in the frequency domain were performed by Doliwa et al. $[10,11]$ and Niedermayer [12] using staircase FIT, which suffers from slow convergence when curved boundaries are present in the geometry. This is particularly problematic for nonultrarelativistic beams, since the source has to be modeled properly to remove direct (transverse) space charge fields. Macridin et al. [13] presented an approach using the boundary element method (BEM). This approach does not include the space charge impedance

*niedermayer@temf.tu-darmstadt.de

Published by the American Physical Society under the terms of the Creative Commons Attribution 3.0 License. Further distribution of this work must maintain attribution to the author $(s)$ and the published article's title, journal citation, and DOI. since the beam is modeled pointlike. Meshing different material domains is possible, but yet involved to automate. An approach to obtain the longitudinal space charge impedance from the electrostatic potential calculated by finite element nodal shape functions is presented by $\mathrm{Li}$ and Wang [14].

This paper presents a general 2D longitudinal and transverse impedance computation tool for arbitrary frequency and beam velocity. It is based on the finite element method (FEM) on an unstructured triangular mesh. This means that the mesh can be adapted to resolve field penetration depths in different materials. Also, a surface impedance boundary condition (SIBC) is included, to avoid meshing extremely small skin depths in good conductors at high frequency. The full Maxwell equations are solved, for an assumed infinitely long structure of arbitrary transverse geometry.

The algorithm works as follows: In order to properly represent discontinuities on material interfaces, the electric vector field in frequency domain is represented by vector valued, tangentially continuous edge functions. These edge functions of lowest order are incapable of representing the irrotational part of the electric field. Therefore, a Helmholtz split is applied and those field components are obtained separately by solving the Poisson equation for the complex valued scalar potential with nodal functions. The impedance is finally obtained from the sum of the solenoidal and irrotational field solutions by a post-processing functional.

The implementation is done using the open source finite element toolbox FEniCS [15] (see also [16]). It provides a mathematical framework to work with function spaces that have been created on the mesh. The PYTHON source code is compiled just in time (JIT) to obtain $\mathrm{C}++$ code that runs a powerful linear algebra backend such as PETSc [17]. FEniCS does not provide complex numbers, but this drawback is overcome by coupling multiple function 
spaces together. The mesh originates from GMSH [18] and it is imported using DOLFIN-CONVERT [19]. Since only open source software is involved, we decided to publish our code as well. It can be downloaded from [20].

The paper is structured as follows: In Sec. II the beam coupling impedance definition is given and the problem is stated. Section III describes how Maxwell's equations are recast to obtain a weak formulation suited to 2D FEM computation. In Sec. IV the solver is benchmarked followed by the conclusion in Sec. V.

\section{PROBLEM STATEMENT}

The beam with total charge $q$ is modeled as a disc with radius $a$ and surface charge density $\sigma$, traveling with velocity $\vec{v}=\beta c \vec{e}_{z}$. The displacement $d_{x}, d_{y}$ of the beam in $x$ or $y$ direction (i.e., a coherent dipole oscillation) can be approximated in cylindrical coordinates $(\varrho, \varphi)$ by

$\sigma(\varrho, \varphi) \approx \frac{q}{\pi a^{2}}\left[\Theta(a-\varrho)+\delta(a-\varrho)\left(d_{x} \cos \varphi+d_{y} \sin \varphi\right)\right]$,

where $\Theta$ and $\delta$ denote the Heaviside step and Dirac $\delta$-function, respectively. The current density reads in the time domain

$$
\vec{J}_{s}(t)=\sigma \delta(z-v t) \vec{v}
$$

resulting in frequency domain as

$$
\begin{aligned}
\overrightarrow{\vec{J}}_{s}(\omega) & =\int_{-\infty}^{\infty} \vec{J}_{s}(t) e^{-i \omega t} \mathrm{~d} t=\sigma e^{-i \omega z / \beta c} \vec{e}_{z} \\
& =\overrightarrow{\underline{J}}_{\|}(\omega)+\underline{\vec{J}}_{d_{x}}(\omega)+\underline{\vec{J}}_{d_{y}}(\omega) .
\end{aligned}
$$

The coherent force acting back on the beam is described by the coupling impedance [2]

$$
\begin{gathered}
\underline{Z}_{\|}(\omega)=-\frac{1}{q^{2}} \int_{\text {beam }} \underline{\vec{E}} \cdot \underline{\vec{J}}_{\|}^{*} \mathrm{~d} V \\
\underline{Z}_{\perp, x}(\omega)=-\frac{\beta c}{\left(q d_{x}\right)^{2} \omega} \int_{\text {beam }} \underline{\vec{E}} \cdot \underline{\vec{J}}_{d_{x}}^{*} \mathrm{~d} V \\
\underline{Z}_{\perp, y}(\omega)=-\frac{\beta c}{\left(q d_{y}\right)^{2} \omega} \int_{\text {beam }} \underline{\vec{E}} \cdot \underline{\vec{J}}_{d_{y}}^{*} \mathrm{~d} V,
\end{gathered}
$$

i.e., the beam's power loss into the particular azimuthal mode. Note that $\underline{\vec{E}}$ and $\underline{\vec{J}}$ are to be understood as spectral densities in units of $\mathrm{V} / \mathrm{m} / \mathrm{Hz}$ and $\mathrm{A} / \mathrm{m}^{2} / \mathrm{Hz}$, respectively.

Since the impedance as given in Eqs. (4)-(6) describes coherent effects, it depends only marginally on the beam shape [21]. Therefore we use the uniform beam distribution as given in Eq. (1). For particular cases, such as the longitudinal space charge impedance, direct and indirect effects cannot be decoupled. Then it makes sense to use the real beam distribution, e.g., a Gaussian transverse profile. Also, the excitation can be extended to higher multipoles by substituting $\varphi \rightarrow m \varphi$ in Eq. (1). Evaluations of the fields at particular points in space, as necessary for a direct evaluation of the detuning impedance as defined in, e.g., [22], are numerically not robust and therefore not attempted here.

The task is to solve Maxwell's equations subject to excitation by Eq. (3), including the charge that can be obtained by the continuity equation. The impedance is then evaluated by Eqs. (4), (5), and (6), interpreted as functionals of the solution of Maxwell's equations.

\section{2D IMPEDANCE FEM SOLVER}

The electric field $\underline{\vec{E}}: \mathbb{R}^{2} \rightarrow \mathbb{C}^{3}$ required to determine the coupling impedance is the solution of

$$
\nabla \times \underline{\nu} \nabla \times \underline{\vec{E}}-\omega^{2} \underline{\varepsilon} \underline{\vec{E}}=-i \omega \underline{\vec{J}}_{s}
$$

with the complex valued reluctivity $\underline{\nu}=\mu^{-1}=$ $\left(\mu^{\prime}+i \mu^{\prime \prime}\right) /|\underline{\mu}|^{2}$, and the complex valued permittivity $\underline{\varepsilon}=$ $\varepsilon_{0} \varepsilon_{r}-i \kappa / \omega$ (conductivity $\kappa$ ) as functions of position and frequency. On the sufficiently smooth boundary $\partial \Omega$ of the computational domain $\Omega \subset \mathbb{R}^{2}$, see Fig. 1, a Dirichlet condition, i.e., $\vec{n} \times \vec{E}=0$, is applied. Later, this will be replaced by a metallic surface impedance boundary condition, relating the electric and magnetic field in the two directions tangential to the boundary.

For a two-dimensional (infinitely long) structure, the Fourier correspondence $\partial_{z} \rightarrow-i \omega / v$, arising from Eq. (3), motivates splitting the electric field as

$$
\overrightarrow{\underline{E}}=\left(\begin{array}{c}
\vec{E}_{\perp}^{\mathrm{r}} \\
E_{z}^{\mathrm{r}}
\end{array}\right)+i\left(\begin{array}{c}
\vec{E}_{\perp}^{\mathrm{i}} \\
E_{z}^{\mathrm{i}}
\end{array}\right)
$$

where the upper indices denote real and imaginary parts. If the vectorial (transverse) parts of the field would be discretized using nodal elements, a jump of the normal field component on a material interface would be impossible. In order to avoid this "too much continuity" phenomenon, Nédélec edge-elements [23,24] are used for the vector fields.

Since the lowest order Nédélec elements are not suited to compute the divergence of a field, the irrotational part is calculated separately by applying a Helmholtz split $\underline{\vec{E}}=$ $\vec{E}_{\text {curl }}+\vec{E}_{\text {div }}$ to Eq. (8) on the simply connected domain $\Omega$. Demanding $\nabla \cdot \underline{\varepsilon}_{\text {curl }}=0$ and $\nabla \times \underline{\vec{E}}_{\text {div }}=0$ determines the two uniquely (see [25], p. 86, and p. 170 for the discrete version). From the ansatz $\vec{E}_{\mathrm{div}}=-\nabla \underline{\Phi}$ the Poisson equation for the complex potential is found, 


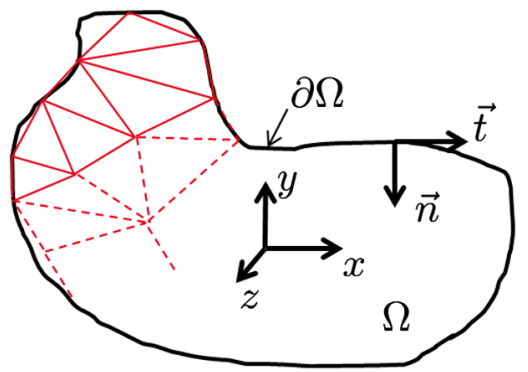

FIG. 1. Computational domain for the 2D impedance solver.

$$
-\nabla \cdot \underline{\varepsilon} \nabla \underline{\Phi}=\underline{\varrho}_{s}=\frac{1}{\beta c} \underline{J}_{s, z}
$$

It represents a coupled electrostatic and stationary current problem. For the solenoidal part the same curl-curl equation as Eq. (7),

$$
\nabla \times \underline{\nu} \nabla \times \underline{\vec{E}}_{\mathrm{curl}}-\omega^{2} \underline{\varepsilon}_{\mathrm{E}} \overrightarrow{\mathrm{curl}}_{\mathrm{l}}=\underline{\vec{R}}
$$

is found, but with a divergence free right-hand side

$$
\underline{\vec{R}}=\omega^{2} \underline{\varepsilon \vec{E}}_{\mathrm{div}}-i \omega \vec{J}_{s},
$$

as visible from the continuity equation.

The continuous fields are elements of their respective Sobolev-spaces (see, e.g., $[25,26]$ ), i.e., $\underline{E}_{z, \text { div } / \text { curl }}^{\mathrm{r}} \in \mathcal{H}^{1}(\Omega)$ and $\underline{\underline{E}}_{\perp, \text { div } / \text { curl }}^{\mathrm{r} / \mathrm{i}} \in \mathcal{H}_{2 \mathrm{D}}^{\text {curl }}(\Omega)$ and they feature the respective properties of the De Rham complex shown in Fig. 2. The unidirectional solid arrows denote complete De Rham sequences, i.e., the range of one operator is the kernel of the next. By definition the Hodge operators map between a space and its dual. Under the given conditions this dual space of $k$-forms is isomorphic to the space of $n-k$-forms (see, e.g., [27], p. 322), where $n=2,3$ is the dimension of the space.

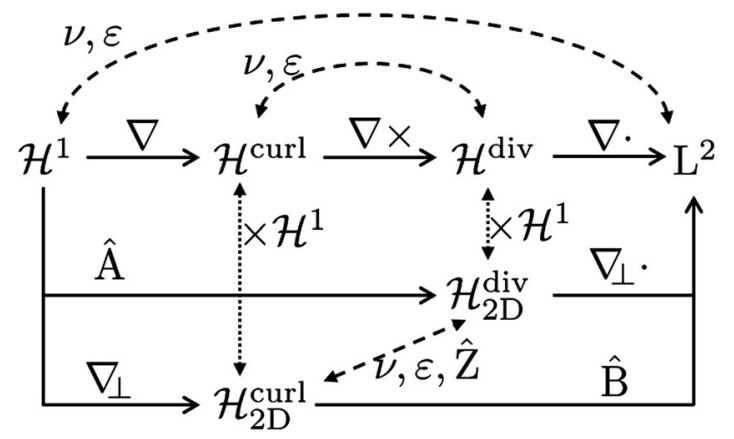

FIG. 2. De Rham diagram for the used operators and function spaces. The operators $\hat{A}: \mathcal{H}^{1}(\Omega) \rightarrow \mathcal{H}_{2 \mathrm{D}}^{\text {div }}(\Omega)$ and $\hat{B}: \mathcal{H}_{2 \mathrm{D}}^{\text {curl }}(\Omega) \rightarrow L^{2}(\Omega)$ are the $2 \mathrm{D}$ vectorial and scalar curl operators, respectively, and $\nu, \varepsilon, \hat{Z}$ are invertible Hodge operators. The $2 \mathrm{D}$ spaces are supplemented by $\mathcal{H}^{1}$ to obtain their $3 \mathrm{D}$ $z$-periodic equivalents.
The same De Rham sequences hold for the discrete fields, provided the function spaces for discrete fields are projections from the continuous function spaces with projection operators that commute with the exterior derivative (see [28], Theorem 5.6).

The functions in $\mathcal{H}^{1}(\Omega)$ are discretized using first order nodal elements

$N_{k}(\xi, \eta)=a_{k} \xi+b_{k} \eta+c_{k}, \quad N_{k}\left(\xi_{i}, \eta_{i}\right)=\delta_{i k}$,

with $i$ and $k$ being local vertex indices and $(\xi, \eta)$ being local coordinates. Transformed to the global coordinates $(x, y)$, these elements are a basis of $\mathcal{H}_{h}^{1}(\Omega)$, where $h$ is the mesh size parameter. For the discretization of $\mathcal{H}_{2 \mathrm{D}}^{\text {curl }}(\Omega)$ functions, we employ lowest order Nédélec edge elements of the first kind (see, e.g., $[16,23]$ ), obtained from the nodal elements by (see, e.g., [29])

$$
\vec{w}_{i}(\xi, \eta)=N_{k} \nabla_{\perp} N_{l}-N_{l} \nabla_{\perp} N_{k},
$$

where $i, k, l$ are again local vertex indices. The edge functions $\vec{w}_{i}$ fulfill

$$
\frac{1}{\left|l_{j}\right|} \int_{l_{j}} \vec{w}_{i} \cdot \vec{t}_{j} \mathrm{~d} s=\delta_{i j}
$$

with $\vec{t}_{j}$ being the tangential unit vector of edge $l_{j}$, which is located at the opposite of node $j$. Transformed to the global coordinates $(x, y)$, the functions in Eq. (13) are a basis of the reduced space $\left\{\vec{u} \in \mathcal{H}_{2 \mathrm{D} h}^{\text {curl }}: \nabla_{\perp} \cdot \vec{u}=0\right\}$, where the divergence operator is to be understood in the discrete weak sense.

\section{A. Source terms}

The source current, Eq. (3), at $z=0$ is projected on the nodal functions by writing it in the $\mathcal{H}_{h}^{1}(\Omega)$-basis as

$$
\sigma(x, y)=\sum_{i=1}^{N_{n}} a_{i}^{\mathrm{r}} N_{i}(x, y) .
$$

The coefficients $a_{i}^{r}$ are obtained by multiplying with $N_{j}$, integrating, and solving the positive definite system for the "mass-matrix"

$$
\mathbf{M}_{i j}=\int_{\Omega} N_{i} N_{j} \mathrm{~d} \Omega
$$

For the dipolar terms the $\delta$-function in Eq. (1) will always be smeared out over a mesh cell. This error can be minimized by adapting the mesh such that a triangular representation of the $\delta$-function, 


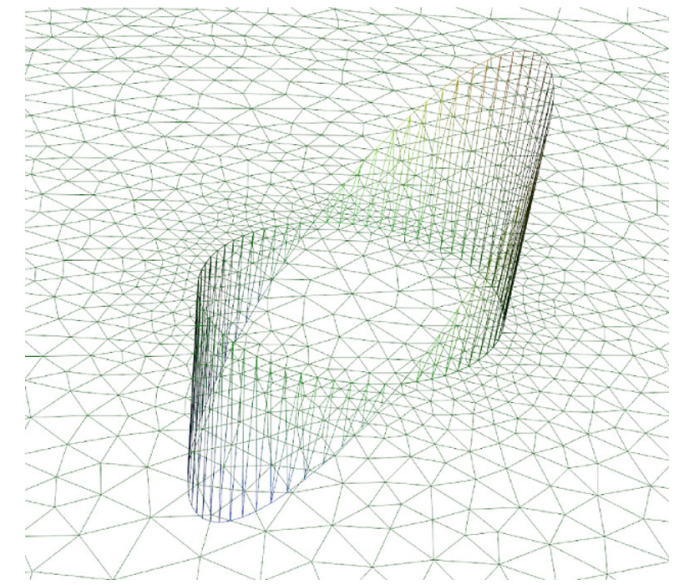

FIG. 3. Horizontal dipole source term $\sigma(x, y)$ in arbitrary units.

$$
\begin{aligned}
\delta_{s}\left(x-x_{0}\right)=\frac{1}{s^{2}}( & \left\{\Theta\left[x-\left(x_{0}-s\right)\right]-\Theta\left[x-x_{0}\right]\right\} \\
& \times\left[x-\left(x_{0}-s\right)\right] \\
-\left\{\Theta\left[x-x_{0}\right]-\right. & \left.\left.\Theta\left[x-\left(x_{0}+s\right)\right]\right\} \times\left[x-\left(x_{0}+s\right)\right]\right),
\end{aligned}
$$

is achieved, with $s \ll a$ being the width of the triangle. Note that $\delta_{s} \in L^{2}$ while $\delta \notin L^{2}$. A plot of this source term can be seen in Fig. 3. The smear-out error can be estimated by calculating the dipole moment of a source [Eq. (1)] represented on the mesh by Eq. (17), i.e.,

$$
\int_{\Omega} \sigma_{d_{x}, s} x \mathrm{~d} x \mathrm{~d} y=q d_{x}\left(1+\frac{1}{6} \frac{s^{2}}{a^{2}}\right)
$$

The required accuracy, also referring to the removal of the direct space charge impedance, is discussed in Sec. IVA.

\section{B. Poisson solver}

The coupled Poisson system Eq. (9) is rewritten in $2 \mathrm{D}$ as

$-\nabla_{\perp} \cdot \varepsilon_{0} \varepsilon_{r} \nabla_{\perp} \Phi^{r}+\frac{\omega^{2} \varepsilon_{0} \varepsilon_{r}}{\beta^{2} c^{2}} \Phi^{r}-\nabla_{\perp} \cdot \frac{\kappa}{\omega} \nabla_{\perp} \Phi^{\mathrm{i}}+\frac{\omega \kappa}{\beta^{2} c^{2}} \Phi^{\mathrm{i}}=\varrho_{s}^{r}$
$-\nabla_{\perp} \cdot \varepsilon_{0} \varepsilon_{r} \nabla_{\perp} \Phi^{\mathrm{i}}+\frac{\omega^{2} \varepsilon_{0} \varepsilon_{r}}{\beta^{2} c^{2}} \Phi^{\mathrm{i}}+\nabla_{\perp} \cdot \frac{\kappa}{\omega} \nabla_{\perp} \Phi^{r}-\frac{\omega \kappa}{\beta^{2} c^{2}} \Phi^{r}=0$.

It is solved by means of the standard Galerkin procedure, i.e., testing the real and imaginary parts of the equation separately with $N_{j}$ and integration by parts to obtain algebraic equations for the real and imaginary coefficients of $\underline{\Phi}$, separately. For example, the first "stiffness" term reads

$$
\begin{aligned}
& -\int_{\Omega} N_{j} \nabla_{\perp} \cdot \varepsilon_{0} \varepsilon_{r} \nabla_{\perp} \Phi^{r} \mathrm{~d} \Omega \\
& =\int_{\Omega} \nabla_{\perp} N_{j} \cdot \varepsilon_{0} \varepsilon_{r} \nabla_{\perp} \Phi^{r} \mathrm{~d} \Omega+\int_{\partial \Omega} N_{j} \vec{n} \cdot \varepsilon_{0} \varepsilon_{r} \nabla_{\perp} \Phi^{r} \mathrm{~d} s
\end{aligned}
$$

with a vanishing boundary term for perfectly electric and also for a metallic surface impedance boundary condition. The first stiffness and mass matrices are

$$
\begin{gathered}
\left(\mathbf{S}_{\varepsilon}^{\mathrm{rr}}\right)_{i j}=\int_{\Omega} \varepsilon_{0} \varepsilon_{r} \nabla_{\perp} N_{i} \cdot \nabla_{\perp} N_{j} \mathrm{~d} \Omega \\
\left(\mathbf{M}_{\varepsilon}^{\mathrm{rr}}\right)_{i j}=\frac{\omega^{2}}{\beta^{2} c^{2}} \int_{\Omega} \varepsilon_{0} \varepsilon_{r} N_{i} N_{j} \mathrm{~d} \Omega
\end{gathered}
$$

such that the whole system becomes

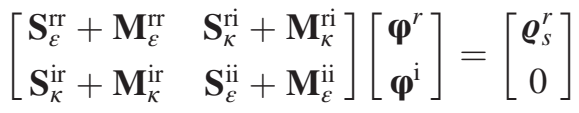

with the bold small letters denoting coefficient vectors.

After solving Eq. (23), the electric field is obtained by $\vec{E}_{\perp, j}^{\mathrm{r}}=\left(\Phi_{k^{\prime}}^{\mathrm{r}}-\Phi_{k}^{\mathrm{r}}\right) \vec{t}_{j} / l_{j}$, where $k$ and $k^{\prime}$ are the nodes attached to the edge $l_{j}$. It is projected, in the same manner as for the source, on the $\mathcal{H}_{2 \mathrm{D} h}^{\text {curl }}(\Omega)$ basis. Together with $E_{z}^{\mathrm{r}}=-\frac{\omega}{\beta c} \Phi^{\mathrm{i}}$ and the respective imaginary parts, the source term for the curl-curl solver, Eq. (11), is assembled.

\section{Curl-curl solver}

In order to solve Eq. (10) the curl operator is decomposed as

$\nabla \times \underline{\vec{E}}=\left(\begin{array}{cc|c}0 & -\partial_{z} & \partial_{y} \\ \partial_{z} & 0 & -\partial_{x} \\ \hline-\partial_{y} & \partial_{x} & 0\end{array}\right) \underline{\vec{E}}=:\left(\begin{array}{l|l}i \hat{\mathrm{Z}} & \hat{\mathrm{A}} \\ \hline \hat{\mathrm{B}} & 0\end{array}\right) \overrightarrow{\underline{E}}$.

The two-dimensional vectorial and scalar curl operators are concatenations of $\hat{\mathrm{A}}_{3 \mathrm{D}}=-\vec{e}_{z} \times \nabla$ and $\hat{\mathrm{B}}_{3 \mathrm{D}}=\vec{e}_{z} \cdot \nabla \times$ and have the property

$$
\hat{\mathrm{A}}=\left(\begin{array}{c}
\partial_{y} \\
-\partial_{x}
\end{array}\right)=-\hat{\mathrm{B}}^{T}
$$

The Hodge operator $\hat{Z}$ is a concatenation of $i \hat{Z}_{3 \mathrm{D}}=$ $\partial_{z} \vec{e}_{z} \times=-i \omega /(\beta c) \vec{e}_{z} \times$ and has the property

$$
\hat{\mathrm{Z}}^{2}=-\frac{\omega^{2}}{\beta^{2} c^{2}} \hat{\mathrm{I}}
$$


The curl-curl term in Eq. (10) is then rewritten as

$$
\begin{aligned}
\nabla \times \underline{\nu} \nabla \times \underline{\vec{E}}= & \left(\begin{array}{cc}
\hat{\mathrm{A}} \nu^{r} \hat{\mathrm{B}}-\nu^{r} \hat{\mathrm{Z}}^{2} & -\hat{\mathrm{Z}} \nu^{\mathrm{i}} \hat{\mathrm{A}} \\
-\hat{\mathrm{B}} \nu^{\mathrm{i}} \hat{\mathrm{Z}} & \hat{\mathrm{B}} \nu^{r} \hat{\mathrm{A}}
\end{array}\right)\left(\begin{array}{c}
\vec{E}_{\perp}^{r} \\
E_{z}^{r}
\end{array}\right)-\left(\begin{array}{cc}
\hat{\mathrm{A}} \nu^{\mathrm{i}} \hat{\mathrm{B}}-\nu^{\mathrm{i}} \hat{\mathrm{Z}}^{2} & \hat{\mathrm{Z}} \nu^{r} \hat{\mathrm{A}} \\
\hat{\mathrm{B}} \nu^{r} \hat{\mathrm{Z}} & \hat{\mathrm{B}} \nu^{\mathrm{i}} \hat{A}
\end{array}\right)\left(\begin{array}{c}
\vec{E}_{\perp}^{\mathrm{i}} \\
E_{z}^{\mathrm{i}}
\end{array}\right) \\
& +i\left(\begin{array}{cc}
\hat{\mathrm{A}} \nu^{r} \hat{\mathrm{B}}-\nu^{r} \hat{\mathrm{Z}}^{2} & -\hat{\mathrm{Z}} \nu^{\mathrm{i}} \hat{\mathrm{A}} \\
-\hat{\mathrm{B}} \nu^{\mathrm{i}} \hat{\mathrm{Z}} & \hat{\mathrm{B}} \nu^{r} \hat{\mathrm{A}}
\end{array}\right)\left(\begin{array}{c}
\vec{E}_{\perp}^{\mathrm{i}} \\
E_{z}^{\mathrm{i}}
\end{array}\right)+i\left(\begin{array}{cc}
\hat{\mathrm{A}} \nu^{\mathrm{i}} \hat{\mathrm{B}}-\nu^{\mathrm{i}} \hat{\mathrm{Z}}^{2} & \hat{\mathrm{Z}} \nu^{r} \hat{\mathrm{A}} \\
\hat{\mathrm{B}} \nu^{r} \hat{\mathrm{Z}} & \hat{\mathrm{B}} \nu^{\mathrm{i}} \hat{\mathrm{A}}
\end{array}\right)\left(\begin{array}{c}
\vec{E}_{\perp}^{r} \\
E_{z}^{r}
\end{array}\right) .
\end{aligned}
$$

This allows testing Eq. (27) line by line using $\vec{w}_{k}^{\mathrm{r}}, v_{k}^{\mathrm{r}}, \vec{w}_{k}^{\mathrm{i}}, v_{k}^{\mathrm{i}}$ and integrating by parts to obtain a bilinear form, i.e., the curl-curl "stiffness" matrix.

The $\mathbb{R}^{3}$ vectoranalytical identity $\nabla \times(\Phi \vec{w})=\Phi \nabla \times \vec{w}-$ $\vec{w} \times \nabla \Phi$ and Stokes' theorem imply for functions $\varphi: \Omega \rightarrow \mathbb{R}$ and $\vec{u}: \Omega \rightarrow \mathbb{R}^{2}$

$$
\int_{\Omega} \varphi(\hat{\mathrm{B}} \vec{u}) \mathrm{d} \Omega=\int_{\Omega} \vec{u}(\hat{\mathrm{A}} \varphi) \mathrm{d} \Omega-\int_{\partial \Omega} \varphi \vec{u} \cdot \vec{t} \mathrm{~d} s,
$$

where $\vec{t}=\vec{e}_{z} \times \vec{n}$ is the tangential unit vector at the boundary. For the second order operators in Eq. (27) follows

$$
\begin{aligned}
\int_{\Omega}\left(\hat{\mathrm{A}} \nu^{\mathrm{r}} \hat{\mathrm{B}} \vec{E}_{\perp}^{\mathrm{r}}\right) \cdot \vec{w}_{j}^{\mathrm{r}} \mathrm{d} \Omega= & \int_{\Omega}\left(\nu^{\mathrm{r}} \hat{\mathrm{B}} \vec{E}_{\perp}^{\mathrm{r}}\right)\left(\hat{\mathrm{B}} \vec{w}_{j}^{\mathrm{r}}\right) \mathrm{d} \Omega \\
& +\int_{\partial \Omega}\left(\nu^{\mathrm{r}} \hat{\mathrm{B}} \vec{E}_{\perp}^{\mathrm{r}}\right) \vec{w}_{j}^{\mathrm{r}} \cdot \vec{t} \mathrm{~d} s \\
\int_{\Omega}\left(\hat{\mathrm{B}} \nu^{\mathrm{r}} \hat{\mathrm{A}} E_{z}^{\mathrm{r}}\right) v_{j}^{\mathrm{r}} \mathrm{d} \Omega= & \int_{\Omega}\left(\nu^{\mathrm{r}} \hat{\mathrm{A}} E_{z}^{\mathrm{r}}\right) \cdot\left(\hat{\mathrm{A}} v_{j}^{\mathrm{r}}\right) \mathrm{d} \Omega \\
& -\int_{\partial \Omega} v_{j}^{\mathrm{r}}\left(\nu^{\mathrm{r}} \hat{\mathrm{A}} E_{z}^{\mathrm{r}}\right) \cdot \vec{t} \mathrm{~d} s .
\end{aligned}
$$

The boundary terms vanish in case of a Dirichlet (perfectly electric) boundary condition. For a metallic SIBC the relation $\vec{n} \times \vec{n} \times \underline{\vec{E}}=\underline{Z}_{s} \vec{n} \times \underline{\vec{H}}$ with the surface impedance

$$
\underline{Z}_{s}=\frac{1}{\underline{Y}_{s}}=\frac{1+i}{\sqrt{2}} \sqrt{\frac{\omega \mu}{\kappa}}
$$

holds on the boundary $\partial \Omega$. The magnetic field is obtained from Faraday's law as

$$
\begin{gathered}
i \hat{\mathrm{Z}} \underline{E}_{t}+\hat{\mathrm{A}} \underline{E}_{z}=-i \omega \mu \underline{H}_{t}=-i \omega \mu \underline{Y}_{s} \underline{E}_{z}=\underline{k} \underline{E}_{z} \\
\hat{\mathrm{B}} \underline{E}_{t}=-i \omega \mu \underline{H}_{z}=-i \omega \mu \underline{Y}_{s} \underline{E}_{t}=\underline{k} \underline{E}_{t},
\end{gathered}
$$

with the index $t$ denoting the boundary tangential projection and the abbreviation $\underline{k}=-i \omega \mu \underline{Y}_{s}=(-1-i) / \delta$ using the skin depth $\delta=\sqrt{2 /(\mu \kappa \omega)}$. Finally the boundary integrals in Eqs. (29) and (30) read

$$
\begin{gathered}
\int_{\partial \Omega}\left(\nu^{\mathrm{r}} \hat{\mathrm{B}} \vec{E}_{\perp}^{\mathrm{r}}\right) \vec{w}_{j}^{\mathrm{r}} \cdot \vec{t} \mathrm{~d} s=\int_{\partial \Omega} \nu^{\mathrm{r}}\left(\underline{k \vec{E}} \underline{\perp}_{\perp} \cdot \vec{t}\right)^{\mathrm{r}}\left(\vec{w}_{j}^{\mathrm{r}} \cdot \vec{t}\right) \mathrm{d} s \\
\int_{\partial \Omega} v_{j}^{\mathrm{r}}\left(\nu^{\mathrm{r}} \hat{\mathrm{A}} E_{z}^{\mathrm{r}}\right) \cdot \vec{t} \mathrm{~d} s=\int_{\partial \Omega} v_{j}^{\mathrm{r}} \nu^{\mathrm{r}}\left(\underline{k E_{z}}\right)^{\mathrm{r}} \mathrm{d} s,
\end{gathered}
$$

plus the respective imaginary parts. Note that the $\hat{Z} \underline{E}_{t}$ term is normal to the boundary and therefore vanishes.

The discretization of Eq. (10) is finally

$$
\left[\mathbf{S}_{\text {curlcurl }}+\mathbf{M}_{\underline{\varepsilon}}+\mathbf{M}_{\mathrm{SIBC}}\right] \mathbf{e}_{\mathrm{curl}}=\mathbf{r}
$$

with the solution vector

$$
\mathbf{e}_{\text {curl }}=\left[\begin{array}{c}
\mathbf{e}_{\perp}^{\mathrm{r}} \\
\mathbf{e}_{\perp}^{\mathrm{i}} \\
\mathbf{e}_{z}^{\mathrm{r}} \\
\mathbf{e}_{z}^{\mathrm{i}}
\end{array}\right] .
$$

The matrices are arranged as

$$
\begin{gathered}
\mathbf{S}_{\text {curlcurl }}=\left[\begin{array}{llll}
\mathbf{S}_{\perp \perp}^{\mathrm{rr}} & \mathbf{S}_{\perp \perp}^{\mathrm{ir}} & \mathbf{S}_{z \perp}^{\mathrm{rr}} & \mathbf{S}_{z \perp}^{\mathrm{ir}} \\
\mathbf{S}_{\perp \perp}^{\mathrm{ri}} & \mathbf{S}_{\perp \perp}^{\mathrm{ii}} & \mathbf{S}_{z \perp}^{\mathrm{ri}} & \mathbf{S}_{z \perp}^{\mathrm{ii}} \\
\mathbf{S}_{\perp z}^{\mathrm{rr}} & \mathbf{S}_{\perp z}^{\mathrm{ir}} & \mathbf{S}_{z z}^{\mathrm{rr}} & \mathbf{S}_{z z}^{\mathrm{ir}} \\
\mathbf{S}_{\perp z}^{\mathrm{ri}} & \mathbf{S}_{\perp z}^{\mathrm{ii}} & \mathbf{S}_{z z}^{\mathrm{ri}} & \mathbf{S}_{z z}^{\mathrm{ii}}
\end{array}\right] \\
\mathbf{M}_{\underline{\varepsilon}}=\left[\begin{array}{ccccc}
\mathbf{M}_{\varepsilon \perp}^{\mathrm{rr}} & \mathbf{M}_{\kappa \perp}^{\mathrm{ir}} & & \\
\mathbf{M}_{\kappa \perp}^{\mathrm{ri}} & \mathbf{M}_{\varepsilon \perp}^{\mathrm{ii}} & & \\
0 & & \mathbf{M}_{\varepsilon z}^{\mathrm{rr}} & \mathbf{M}_{\kappa z}^{\mathrm{ir}} \\
& & \mathbf{M}_{\kappa z}^{\mathrm{ri}} & \mathbf{M}_{\varepsilon z}^{\mathrm{ii}}
\end{array}\right] \\
\mathbf{M}_{\mathrm{SIBC}}=\left[\begin{array}{lllll}
\mathbf{D}_{\perp}^{\mathrm{rr}} & \mathbf{D}_{\perp}^{\mathrm{ir}} & & \\
\mathbf{D}_{\perp}^{\mathrm{ri}} & \mathbf{D}_{\perp}^{\mathrm{ii}} & & \\
& & \mathbf{T}_{z}^{\mathrm{rr}} & \mathbf{T}_{z}^{\mathrm{ir}} \\
& & \mathbf{T}_{z}^{\mathrm{ri}} & \mathbf{T}_{z}^{\mathrm{ii}}
\end{array}\right]
\end{gathered}
$$

where we exemplary write the terms

$$
\left(\mathbf{S}_{\perp \perp}^{\mathrm{rr}}\right)_{i, j}=\int_{\Omega}\left[\left(\nu^{\mathrm{r}} \hat{\mathrm{B}} \vec{w}_{i}^{\mathrm{r}}\right)\left(\hat{\mathrm{B}} \vec{w}_{j}^{\mathrm{r}}\right)-\nu^{\mathrm{r}} \hat{\mathrm{Z}}^{2} \vec{w}_{i}^{\mathrm{r}} \cdot \vec{w}_{j}^{\mathrm{r}}\right] \mathrm{d} \Omega
$$




$$
\begin{aligned}
\left(\mathbf{M}_{\varepsilon \perp}^{\mathrm{rr}}\right)_{i, j} & =-\omega^{2} \int_{\Omega} \varepsilon \vec{w}_{i}^{\mathrm{r}} \cdot \vec{w}_{j}^{\mathrm{r}} \mathrm{d} \Omega \\
\left(\mathbf{D}_{\varepsilon \perp}^{\mathrm{rr}}\right)_{i, j} & =\int_{\partial \Omega} \nu^{\mathrm{r}} \underline{k}^{\mathrm{r}} \vec{w}_{i}^{\mathrm{r}} \cdot \vec{w}_{j}^{\mathrm{r}} \mathrm{d} s \\
\left(\mathbf{T}_{\varepsilon \perp}^{\mathrm{rr}}\right)_{i, j} & =-\int_{\partial \Omega} \nu^{\mathrm{r}} \underline{k}^{\mathrm{r}} v_{i}^{\mathrm{r}} v_{j}^{\mathrm{r}} \mathrm{d} s .
\end{aligned}
$$

For lowest order elements the system of Eq. (36) has a total number of degrees of freedom (dofs) of 2 - (\#nodes + \#edges). In the presence of different materials it is usually ill conditioned and therefore solved with a direct solver, i.e., standard LU (lower upper) decomposition or MUMPS (multifrontal massively parallel sparse direct solver).

\section{Impedance evaluation}

The impedance functionals Eqs. (4), (5), and (6) are evaluated by inserting the discretizations of $\underline{E}_{z}$ and $\underline{J}_{z}$. One obtains a linear form involving the mass matrix Eq. (16),

$\underline{Z}_{\|}=-\frac{l}{q^{2}} \sum_{j, k=1}^{N_{n}}\left(a_{k}^{\mathrm{r}}-i a_{k}^{\mathrm{i}}\right) M_{j k}\left(e_{j}^{\mathrm{r}}+i e_{j}^{\mathrm{i}}\right)=-\frac{l}{q^{2}} \mathbf{a}^{H} \mathbf{M e}$,

with $e_{j}^{\mathrm{r}, \mathrm{i}}$ being the coefficients of the total longitudinal electric field and $l$ being the length of the structure. The transverse impedance is evaluated accordingly, with normalization to the numerically integrated dipole moment, see Eq. (18).

\section{SELECTED BENCHMARK EXAMPLES AND APPLICATIONS}

This section will first show analytically accessible examples, in order to validate the code, and then a real world example, i.e., a collimator. The choice of the mesh depends not only on frequency and materials, but also on $\beta$. A rule of thumb is to take 4 mesh cells per wavelength $\lambda_{\perp}=2 \pi /\left|\underline{k}_{\perp}\right|$ and if necessary perform convergence studies. The transverse wave number $k_{\perp}$ is subject to the dispersion relation

$$
\underline{k}_{\perp}^{2}+\underline{k}_{z}^{2}=\omega^{2} \underline{\mu} \underline{\epsilon}
$$

rewritten with lossless refraction index $n=\sqrt{\mu_{r}^{\prime} \varepsilon_{r}^{\prime}}$ and loss tangents (see also [30]) as

$$
\begin{aligned}
\underline{k}_{\perp}^{2}(x, y)= & \frac{\omega^{2}}{c_{0}^{2}}\left[n^{2}\left(1-\tan \delta_{\mu} \tan \delta_{\varepsilon}\right)\right. \\
& \left.-\frac{1}{\beta^{2}}-i n^{2}\left(\tan \delta_{\mu}+\tan \delta_{\varepsilon}\right)\right] .
\end{aligned}
$$

This indicates, that the mesh requirements can either be dominated by very low $\beta$ or by the material properties.

\section{A. Space charge impedance}

The space charge impedance for a uniform circular beam in a perfectly conducting circular pipe can be obtained from the solution of

$$
\left(\Delta_{\perp}+k_{\varrho}^{2}\right) \underline{E}_{z}=-\frac{\sigma k_{\varrho}^{2}}{i \omega \varepsilon_{0}}
$$

with $\underline{k}_{O}=i \omega /(\beta \gamma c)$ and $\gamma$ being the relativistic mass factor. While for the longitudinal space charge impedance direct and indirect interactions cannot be separated, the transverse space charge impedance requires separation since the direct part acts incoherently on the beam. For a perfectly conducting circular pipe the solutions to Eq. (48) are [2] [Definitions as in Eqs. (4)-(6)]

$$
\begin{aligned}
\underline{Z}_{\|}^{\text {spch }}= & \frac{l}{i \omega \varepsilon_{0} \pi a^{2}}\left[1-2 I_{1}\left(k_{\varrho} a\right)\left(K_{1}\left(k_{\varrho} a\right)\right.\right. \\
& \left.\left.+\frac{K_{0}\left(k_{Q} b\right)}{I_{0}\left(k_{\varrho} b\right)} I_{1}\left(k_{\varrho} a\right)\right)\right] \\
\underline{Z}_{\perp}^{\text {spch }}= & \frac{i l Z_{0}}{\beta \gamma^{2} \pi a^{2}} I_{1}^{2}\left(k_{\varrho} a\right)\left(\frac{K_{1}\left(k_{Q} b\right)}{I_{1}\left(k_{\varrho} b\right)}-\frac{K_{1}\left(k_{\varrho} a\right)}{I_{1}\left(k_{\varrho} a\right)}\right),
\end{aligned}
$$

where $Z_{0}=377 \Omega$ is the vacuum impedance and $I_{n}, K_{n}$ are the modified Bessel functions of first and second kind and order $n$, respectively. The asymptotes for low and high frequency are

$\underline{Z}_{\|, \mathrm{LF}}^{\mathrm{spch}}=\frac{-i \omega \mu_{0} l g}{2 \pi \beta^{2} \gamma^{2}}, \quad g=\frac{1}{4}+\ln \frac{b}{a}, \quad \underline{Z}_{\|, \mathrm{HF}}^{\mathrm{spch}}=\frac{-i l}{\omega \varepsilon_{0} \pi a^{2}}$

$Z_{\perp, \mathrm{LF}}^{\mathrm{spch}}=\frac{-i Z_{0} l}{2 \pi \beta \gamma^{2}}\left(\frac{1}{a^{2}}-\frac{1}{b^{2}}\right), \quad \underline{Z}_{\perp, \mathrm{HF}}^{\mathrm{spch}}=\frac{-i l Z_{0} c}{2 \pi a^{3} \gamma \omega}$.

Figure 4 shows the longitudinal space charge impedance from our code, compared to the analytical one. The

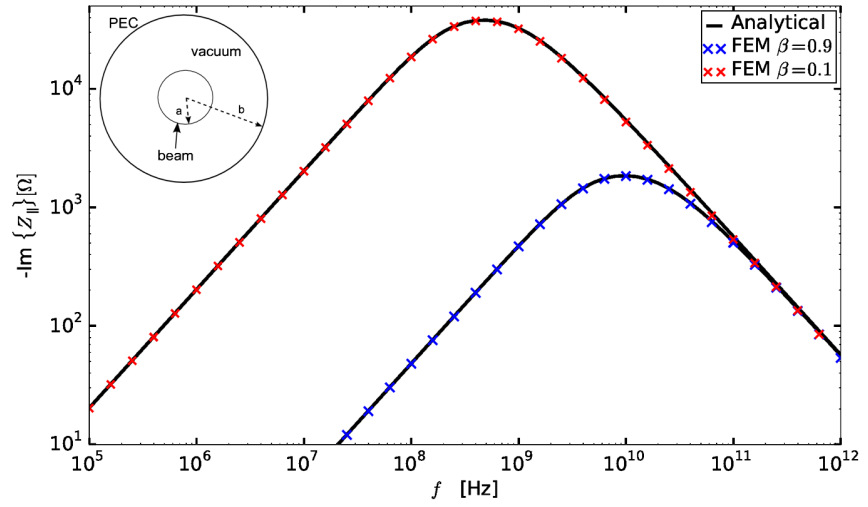

FIG. 4. Longitudinal space charge impedance for a uniform cylindrical beam with radius $a=1 \mathrm{~cm}$, perfectly electric conducting (PEC) pipe with radius $b=4 \mathrm{~cm}$, and length $l=1 \mathrm{~m}$. 


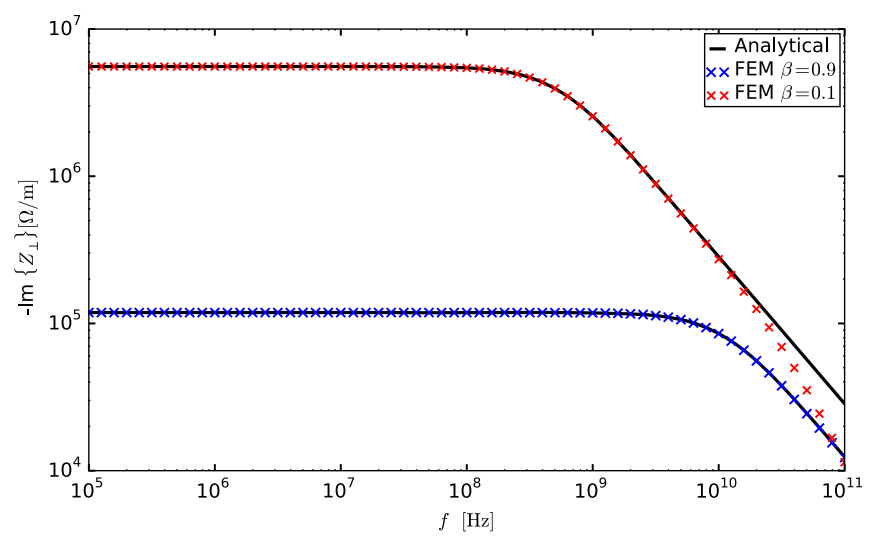

FIG. 5. Transverse space charge impedance (both direct and indirect). The dimensions are the same as in Fig. 4.

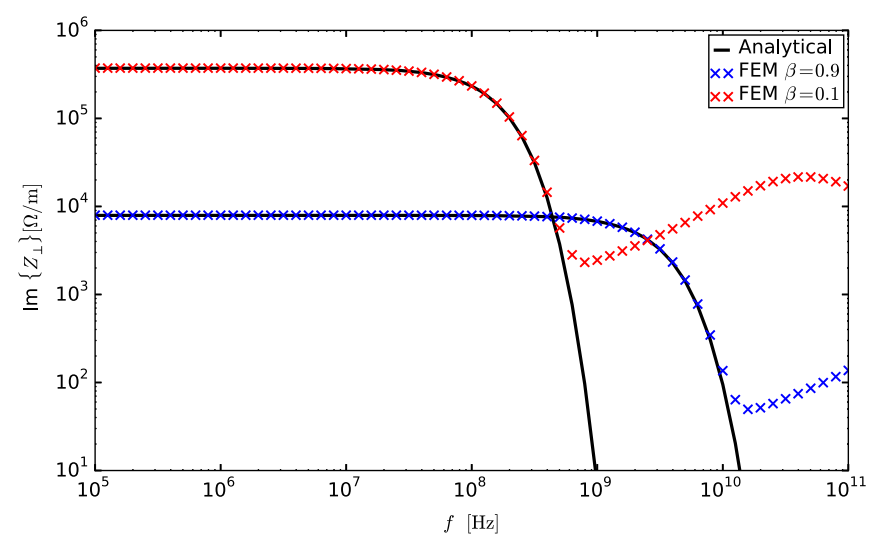

FIG. 6. Transverse space charge impedance (only indirect). The dimensions are the same as in Fig. 4. maximum is at the cutoff frequency $\omega_{c}=\beta \gamma c / a$. The total transverse impedance is visible in Fig. 5. It features the same cutoff frequency. Above cutoff the impedance does not depend on the pipe anymore, i.e., only the direct part remains. Figure 6 shows the indirect transverse space charge impedance that has been obtained by subtracting the analytical direct part from the simulated impedance. Note that for this purpose one requires high accuracy to avoid numerical cancellation (the direct part is by $\approx b^{2} / a^{2}$ larger than the indirect part). Above cutoff the indirect part of the transverse space charge impedance vanishes [see Eq. (52)], therefore the remainder visible in Fig. 6 is just the numerical error. For an accurate calculation at the cutoff frequency, the value for $s$ in Eq. (17) has to be chosen very small, here $s=5 \times 10^{-6} \mathrm{~m}$. Note that a too small $s$ causes triangles with very small angles in the mesh, which deteriorates the condition number of the system matrix.

\section{B. Resistive wall}

The next example is a thin resistive pipe, similar as the one used in the GSI SIS18 and SIS100 synchrotrons, see also [31]. In Fig. 7 one can see the transverse impedance of such a pipe, compared to an analytic calculation by the Mathematica ${ }^{\circledR}$ [8] script Rewall [9]. The curves labeled RW denote a computation where the thin wall is resolved by the mesh. It is valid up to a frequency slightly above the skin effect frequency $f_{\text {skin }}=1 /\left(\pi \mu \kappa d^{2}\right)$. Far above this frequency the mesh cannot resolve the skin depth anymore, therefore the SIBC is applied. For frequencies $f \gtrsim f_{\text {skin }}$,

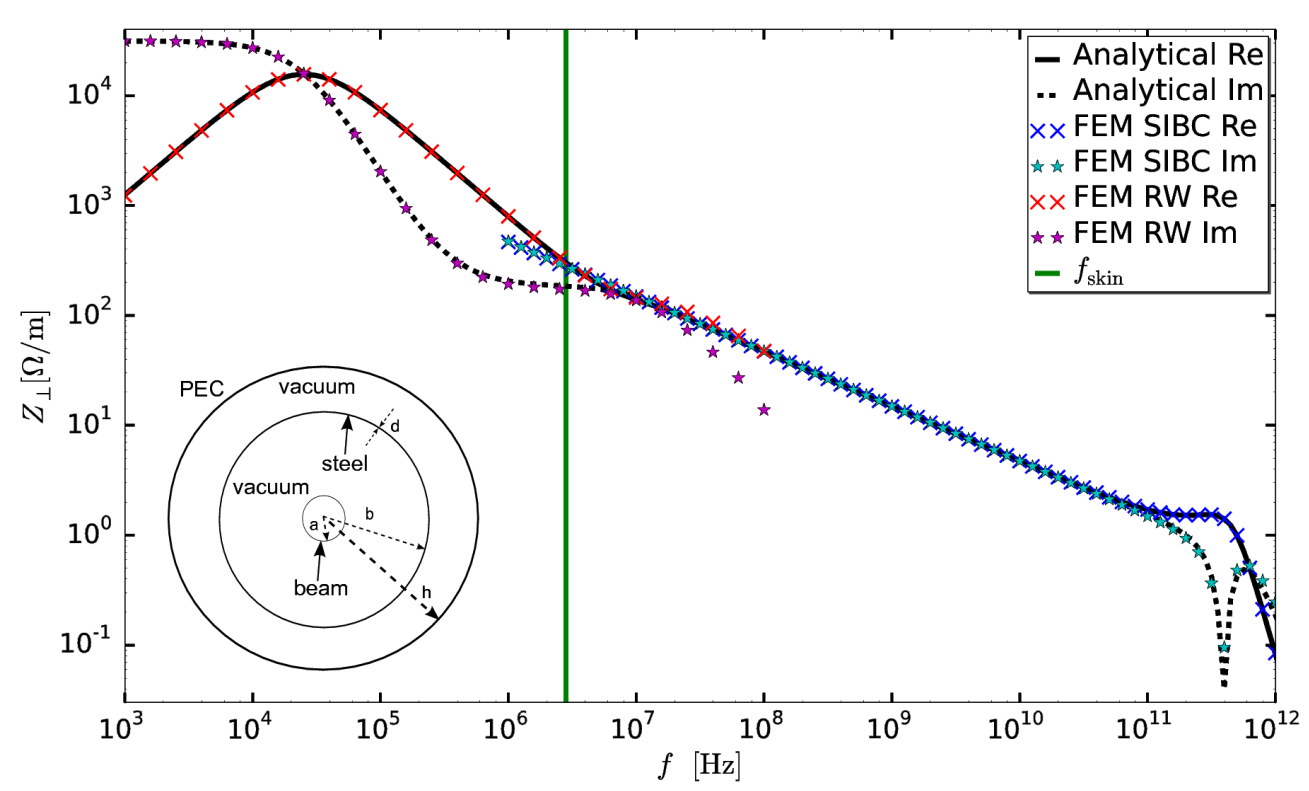

FIG. 7. Transverse impedance of a resistive pipe for $\beta=0.999999$ (beam radius $a=1 \mathrm{~cm}$, pipe radius $b=4 \mathrm{~cm}$, thickness $d=0.3 \mathrm{~mm}, h=1 \mathrm{~m}, l=1 \mathrm{~m}$, conductivity $\kappa=10^{6} \mathrm{~S} / \mathrm{m}$.) 


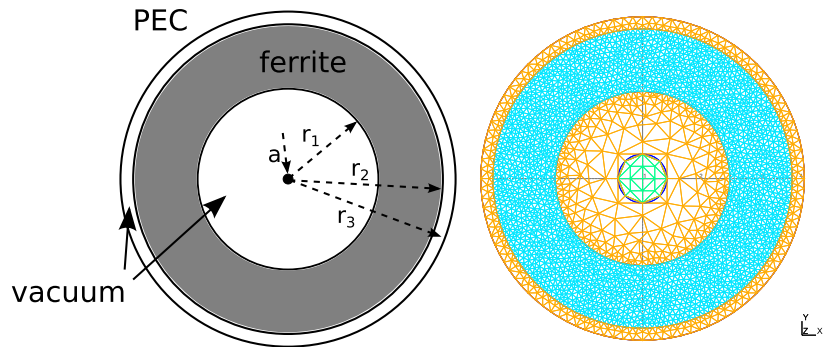

FIG. 8. Ferrite ring geometry and mesh, $a=0.5 \mathrm{~cm}$, $r_{1}=1.78 \mathrm{~cm}, r_{2}=3.05 \mathrm{~cm}, r_{3}=3.3 \mathrm{~cm}, l=2.54 \mathrm{~cm}$.

i.e., $f \approx 10 \mathrm{MHz}$, both solutions have to reproduce the analytical value.

\section{Dispersive Ferrite components}

Our code is also applicable to accelerator components with dispersive materials. Figure 8 shows a ferrite ring made of "Amidon Material 43" [32]. The longitudinal impedance results are visible together with the analytical results in Fig. 9. The analytical results are obtained by a "field matching" algorithm implemented in Mathematica ${ }^{\circledR}$ [8], see [30].

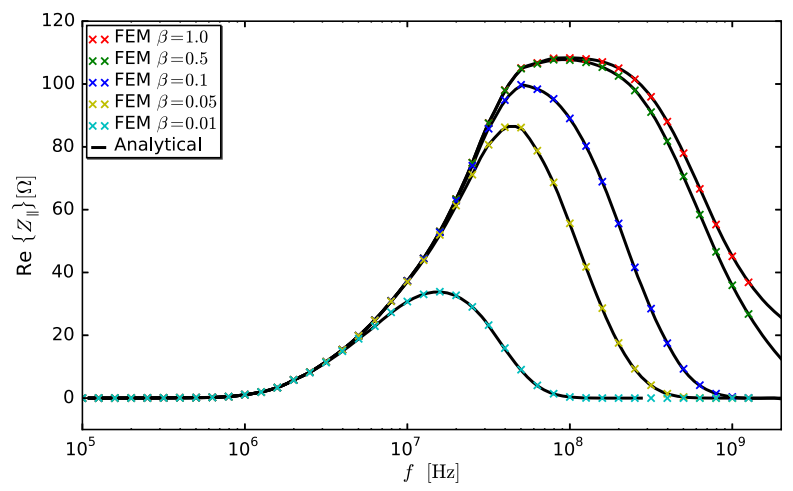

\section{Lossy collimator}

Finally we investigate a lossy carbon $\left(\kappa=10^{4} \mathrm{~S} / \mathrm{m}\right)$ collimator, similar to the ones used in the LHC (see, e.g., $[33,34])$, but simplified. An example shape and the electric field distribution are shown in Fig. 10. Figures 11 and 12 show the longitudinal and transverse impedances for a relativistic $(\beta=0.999999)$ and a nonrelativistic $(\beta=0.1)$ beam. For low frequencies, the jaw material is meshed (RW curve), whereas for high frequency the SIBC is employed. At frequencies where the skin depth is well resolved by the mesh and the skin depth is still much smaller than the jaw thickness, the SIBC and RW curves coincide, as expected.

For $\beta=0.1$ the imaginary part is dominated by the space charge impedance, see again Figs. 4 and 6, where again at high frequency the aforementioned pollution for the indirect transverse space charge impedance occurs. For the real parts we recognize for $\beta=0.1$ the cutoff frequency at $f_{c}=\beta \gamma c /(2 \pi a) \approx 5 \mathrm{GHz}$. Moreover, the longitudinal impedance shows the typical $\propto \sqrt{f}$ behavior, where at $\approx 20 \mathrm{kHz}$ there is a $\operatorname{Re}\left\{Z_{\|}\right\} \propto f^{2}$ edge, due to the image current running on the bounding box instead of the carbon jaws at very low frequency. The transverse

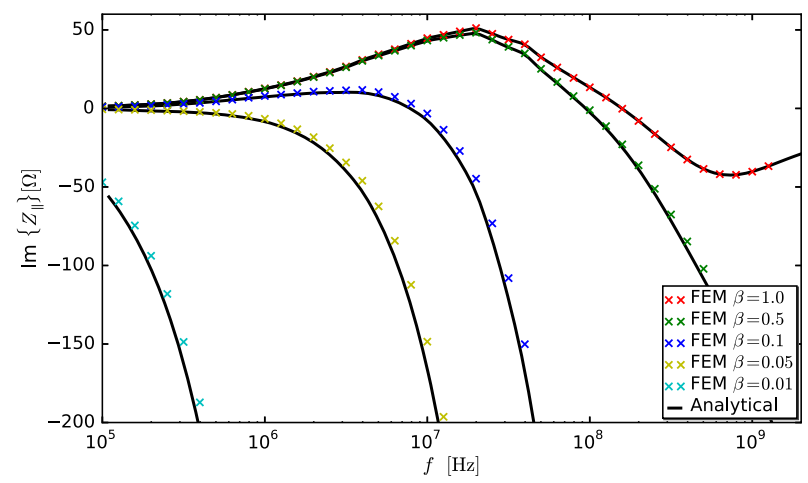

FIG. 9. Longitudinal impedance of a ferrite ring for different beam velocity.
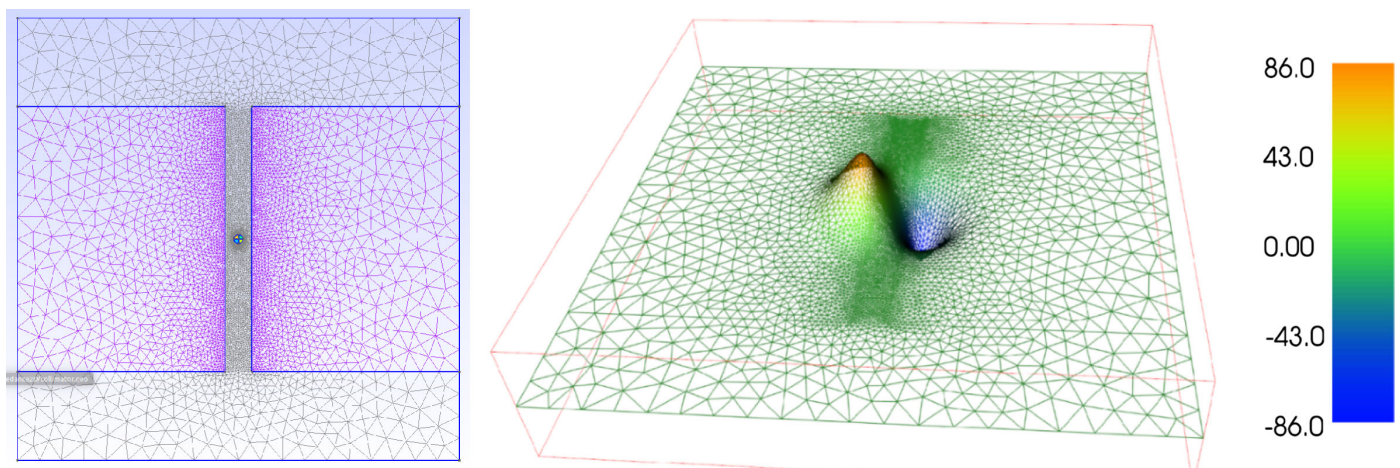

FIG. 10. Resistive collimator with $3 \mathrm{~mm}$ half gap and $3 \mathrm{~cm}$ half jaw height in a $10 \mathrm{~cm} \times 10 \mathrm{~cm}$ bounding box. Mesh from GMSH (left), where the carbon jaws are in magenta and vacuum is in grey. The real part of the longitudinal electric field (in $\mathrm{V} / \mathrm{m} / \mathrm{Hz}$ ) for horizontal dipole excitation at $1 \mathrm{MHz}$ and $\beta=0.1$ is depicted on the right. 

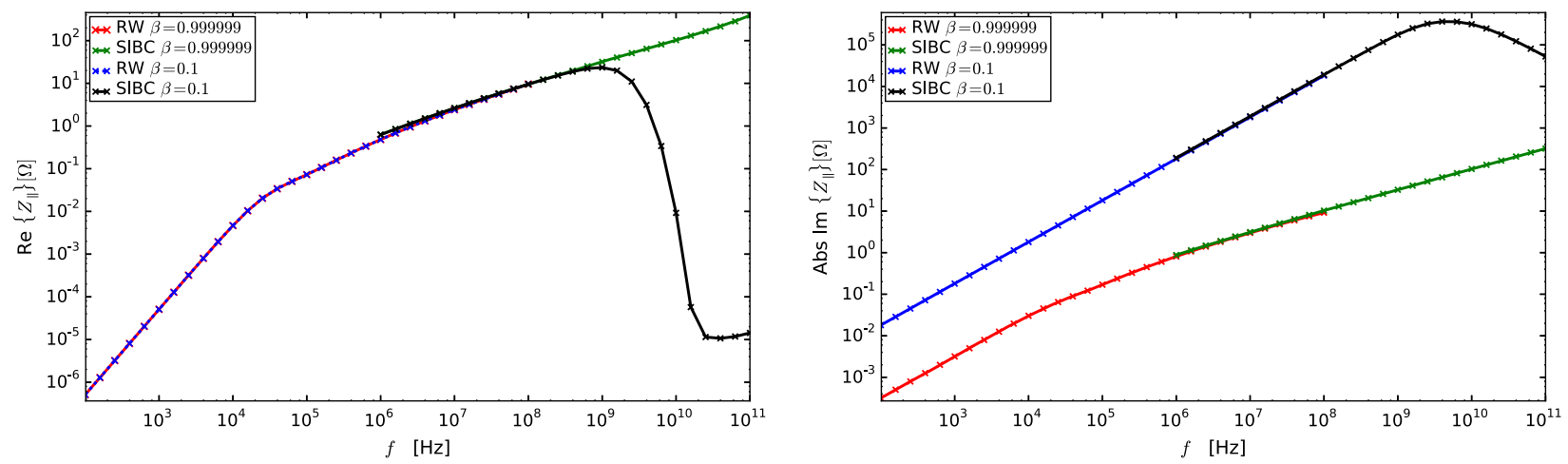

FIG. 11. Longitudinal impedance of the collimator.
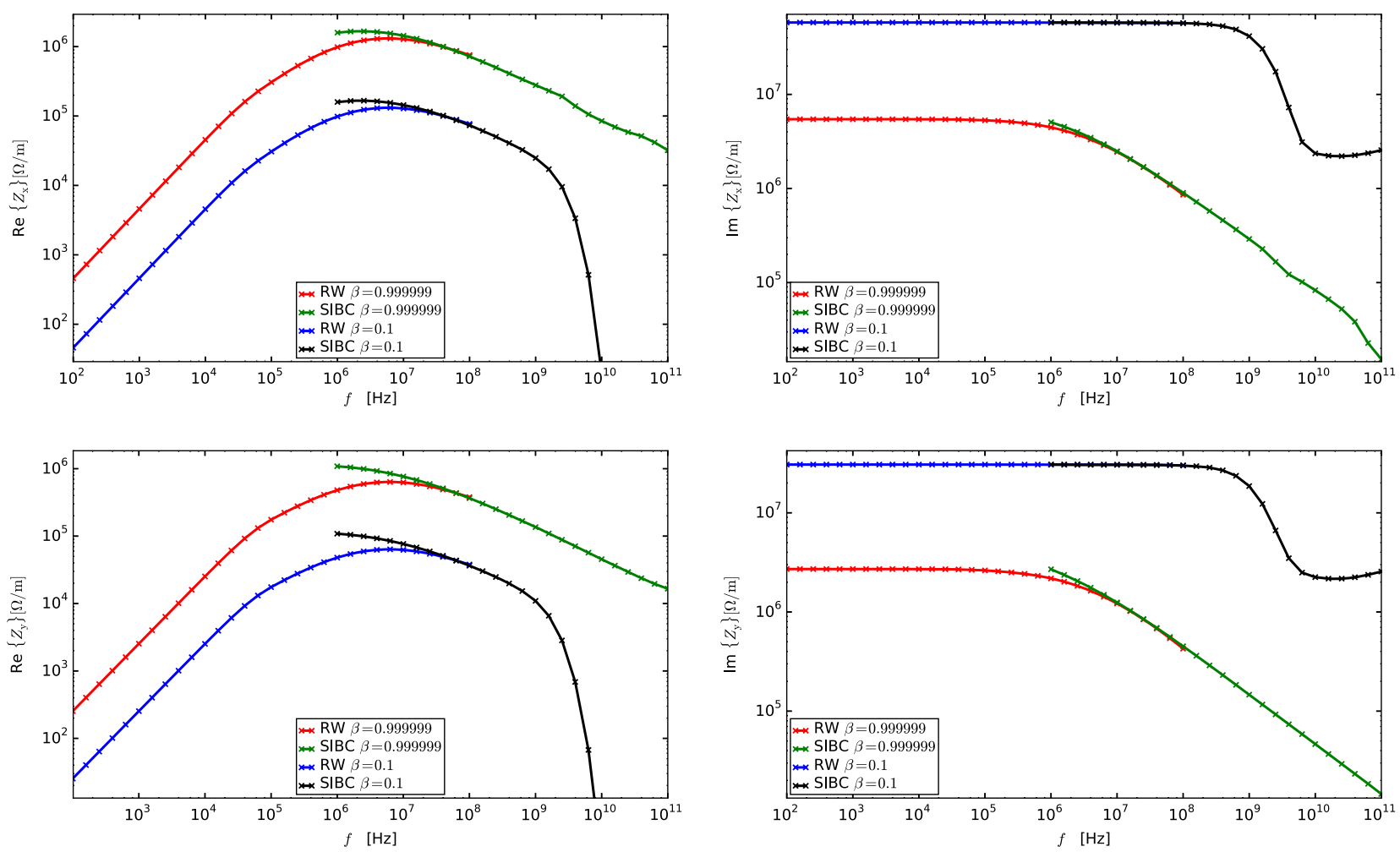

FIG. 12. Transverse impedance (indirect) of the collimator.

impedance shows the dependence as $\operatorname{Re}\left\{Z_{\perp}\right\} \propto$ $f, \operatorname{Im}\left\{Z_{\perp}\right\}=$ const and $Z_{\perp} \propto 1 / \sqrt{f}$ for low and high frequency, respectively, as it has been discussed in more detail in [33] and [34].

\section{CONCLUSION AND OUTLOOK}

A longitudinal and transverse impedance computation tool for arbitrary transverse geometries and material distributions as well as arbitrary frequencies and beam velocities is presented. It is based on the finite element method with Nédélec edge elements. The irrotational part of the electric field is calculated separately, such that the right-hand side of the curl-curl equation becomes divergence free. This ensures the solution to be free of spurious modes while the irrotational fields, necessary for the computation of space charge impedances, are still represented.

The tool was tested for both space charge and resistive wall impedances and showed good agreement with analytical references. Moreover, a lossy collimator has been investigated as an analytically nonaccessible example.

The code runs on usual PCs with the standard LU solver up several $10^{5}$ dofs and with MUMPS up to several $10^{6}$ dofs. The limitation arises rather from memory than from CPU speed. Increasing the approximation order (p-refinement) is only globally possible in FEniCS, which increases the number of dofs significantly. Therefore local mesh refinement (h-refinement) is advantageous in most 
cases. In the future, it is planned to extend the presented FEM algorithm to 3D.

\section{ACKNOWLEDGMENTS}

The authors would like to thank Ulrich Römer and Wolfgang Ackermann for fruitful discussions.

[1] T. Weiland and R. Wanzenberg, Wake fields and impedances, Lect. Notes Phys. 400, 39 (1992).

[2] R. Gluckstern, " Analytic methods for calculating coupling impedances," (CERN Accelerator School, Report No. CERN-2000-011, 2000).

[3] B. Salvant et al., in Proc. of ICFA-HB (2012).

[4] I. Zagorodnov, R. Schuhmann, and T. Weiland, Long-time numerical computation of electromagnetic fields in the vicinity of a relativistic source, J. Comput. Phys. 191, 525 (2003).

[5] I. Zagorodnov and T. Weiland, TE/TM field solver for particle beam simulations without numerical Cherenkov radiation, Phys. Rev. ST Accel. Beams 8, 042001 (2005).

[6] A. Al-Khateeb, O. Boine-Frankenheim, R. Hasse, and I. Hofmann, Longitudinal impedance and shielding effectiveness of a resistive beam pipe for arbitrary energy and frequency, Phys. Rev. E 71, 026501 (2005).

[7] A. Al-Khateeb, R. Hasse, O. Boine-Frankenheim, W. Daqa, and I. Hofmann, Transverse resistive wall impedances and shielding effectiveness for beam pipes of arbitrary wall thickness, Phys. Rev. ST Accel. Beams 10, 064401 (2007).

[8] "Wolfram Mathematica 9.0. www.wolfram.com," (2013).

[9] N. Mounet and E. Metral, Report No. CERN-BE-2009039, 2009.

[10] B. Doliwa, H. De Gersem, T. Weiland, and T. Boonen, Optimised electromagnetic 3D field solver for frequencies below the first resonance, IET Sci. Meas. Technol. 1, 53 (2007).

[11] B. Doliwa, E. Arevalo, and T. Weiland, Numerical calculation of transverse coupling impedances: Comparison to Spallation Neutron Source extraction kicker measurements, Phys. Rev. ST Accel. Beams 10, 102001 (2007).

[12] U. Niedermayer and O. Boine-Frankenheim, in Proceedings of International Computational Accelerator Conference (ICAP), Rostock, Germany, 2012.

[13] A. Macridin, P. Spentzouris, and J. Amundson, Nonperturbative algorithm for the resistive wall impedance of general cross-section beam pipes, Phys. Rev. ST Accel. Beams 16, 121001 (2013).

[14] Y. Li and L. Wang, Space charge impedances of a rectangular beam with longitudinal density modulations inside a rectangular chamber, Nucl. Instrum. Methods Phys. Res., Sect. A 747, 30 (2014).
[15] "FEniCS, www.fenicsproject.org," (2014).

[16] A. Logg, K.-A. Mardal, and G. N. Wells, Automated Solution of Differential Equations by the Finite Element Method (Springer, New York, 2013).

[17] "Portable, Extensible Toolkit for Scientific Computation, http://www.mcs.anl.gov/petsc/," (2014).

[18] C. Geuzaine and J.-F. Remacle, "GMSH 2.8.4 www.geuz .org/gmsh," (2014).

[19] A. Logg, "DOLFIN-CONVERT, www.people.sc.fsu.edu/ jburkardt/py_src/dolfin-convert/dolfin-convert.html," (2010).

[20] "https://bitbucket.org/uniederm/beamimpedance2d/src," (2015).

[21] A. Al-Khateeb, R. W. Hasse, and O. Boine-Frankenheim, Comparison of the longitudinal coupling impedance from different source terms, Nucl. Instrum. Methods Phys. Res., Sect. A 593, 171 (2008).

[22] A. Burov and V. Danilov, Suppression of Transverse Bunch Instabilities by Asymmetries in the Chamber Geometry, Phys. Rev. Lett. 82, 2286 (1999).

[23] J. C. Nedelec, Mixed finite elements in $\mathbb{R}^{3}$, Numer. Math. 35, 315 (1980).

[24] J. C. Nedelec, A new family of mixed finite elements in $\mathbb{R}^{3}$, Numer. Math. 50, 57 (1986).

[25] P. Monk, Finite Element Methods for Maxwell's Equations (Oxford University Press, New York, 2003).

[26] R. A. Adams and J. Fournier, Sobolev Spaces (Elsevier, Amsterdam, 2003).

[27] D. N. Arnold, R. S. Falk, and R. Winther, Finite element exterior calculus: From Hodge theory to numerical stability, Bull. Am. Math. Soc. 47, 281 (2010).

[28] D. N. Arnold, R. S. Falk, and R. Winther, Finite element exterior calculus, homological techniques, and applications, Acta Numer. 15, 1 (2006).

[29] P. Ingelström, A new set of H(curl)-conforming hierarchical basis functions for tetrahedral meshes, IEEE Trans. Microwave Theory Tech. 54, 106 (2006).

[30] U. Niedermayer, L. Eidam, and O. Boine-Frankenheim, Analytic modeling, simulation and interpretation of broadband beam coupling impedance bench measurements, Nucl. Instrum. Methods Phys. Res., Sect. A 776, 129 (2015).

[31] U. Niedermayer and O. Boine-Frankenheim, Analytical and numerical calculations of resistive wall impedances for thin beam pipe structures at low frequencies, Nucl. Instrum. Methods Phys. Res., Sect. A 687, 51 (2012).

[32] "Amidon Material 43, http://www.amidon.de/contents/de/ d542.html," (2013).

[33] T. Kroyer, Report No. CERN-AB-Note-2008-017, 2008.

[34] F. Roncarolo, F. Caspers, T. Kroyer, E. Métral, N. Mounet, B. Salvant, and B. Zotter, Comparison between laboratory measurements, simulations, and analytical predictions of the transverse wall impedance at low frequencies, Phys. Rev. ST Accel. Beams 12, 084401 (2009). 